\title{
Structural and morphological properties of ITO thin films grown by magnetron sputtering
}

\author{
Z. Ghorannevis ${ }^{1}$ E. Akbarnejad ${ }^{2}$ M. Mhoranneviss ${ }^{2}$
}

Received: 8 June 2015/Accepted: 15 August 2015/Published online: 7 October 2015

(c) The Author(s) 2015. This article is published with open access at Springerlink.com

\begin{abstract}
Physical properties of transparent and conducting indium tin oxide (ITO) thin films grown by radiofrequency (RF) magnetron sputtering are studied systematically by changing deposition time. The X-ray diffraction (XRD) data indicate polycrystalline thin films with grain orientations predominantly along the $\left(\begin{array}{lll}2 & 2 & 2\end{array}\right)$ and $\left(\begin{array}{lll}4 & 0 & 0\end{array}\right)$ directions. From atomic force microscopy (AFM) it is found that by increasing the deposition time, the roughness of the film increases. Scanning electron microscopy (SEM) images show a network of a high-porosity interconnected nanoparticles, which approximately have a pore size ranging between 20 and $30 \mathrm{~nm}$. Optical measurements suggest an average transmission of $80 \%$ for the ITO films. Sheet resistances are investigated using fourpoint probes, which imply that by increasing the film thickness the resistivities of the films decrease to $2.43 \times 10^{-5} \Omega \mathrm{cm}$.
\end{abstract}

Keywords Indium tin oxide - Magnetron sputtering · Thickness

\section{Introduction}

Transparent conducting oxide (TCO) thin films are used for various optoelectronics applications such as plasma displays, solar cells, and organic light-emitting diodes. TCO

\section{Z. Ghorannevis}

ghoranneviszohreh@gmail.com

1 Department of Physics, Karaj Branch, Islamic Azad University, Karaj, Iran

2 Plasma Physics Research Centre, Science and Research Branch, Islamic Azad University, Tehran, Iran thin films are grown with different materials such as zinc oxide, tin oxide, indium tin oxide and cadmium tin oxide, as they possess unique electrical and optical properties including good conductivity (about $10^{4} \Omega^{-1} \mathrm{~cm}^{-1}$ ) and high transmittance ( $85 \%$ ) in the visible region due to its large band gap of about $3.70 \mathrm{eV}[1,2] . \mathrm{In}_{2} \mathrm{O}_{3}: \mathrm{Sn}$ (also called indium tin oxide or ITO) is a well-known TCO. Asgrown ITO films are usually highly degenerate n-type semiconductors due to their large number of oxygen vacancies as well as substitution Sn dopants [3-5].

The physical properties of ITO films such as microstructure, electrical and optical properties depend on the deposition methods and experimental conditions. Various growth conditions may affect the crystalline, impurity levels and surface roughness as well as the band gap of the grown thin films [3, 6]. Various techniques, such as electron beam evaporation [7], ion beam-assisted deposition [8], pulsed laser ablation [9, 10], ion implantation [11] and RF/DC magnetron sputtering [1214], are applied for deposition of ITO thin films. Besides the process parameters, the thickness also can affect the properties of the samples [15-22].

In this study, we report the physical properties of ITO thin films prepared via RF sputtering. The sputtering system used in these experiments is a homemade setup and can simultaneously accommodate up to four targets, which makes it an ideal setup for developing thin-film solar cells, without breaking the high vacuum. The substrates can be rotated to be placed under the next target and different layers can be deposited in a high vacuum one by one. The effects of film thickness on the properties of the ITO films were studied to obtain the optimum growth condition to fabricate $\mathrm{CdTe} / \mathrm{CdS}$ thin-film solar cells. Still, it is a challenge to find an optimum condition to improve both optical and electrical properties which are the fundamental aspects for TCO layers. 
ITO thin films are characterized by X-ray diffraction (XRD), scanning electron microscopy (SEM), atomic force microscopy (AFM), spectrophotometer and four-point probe.

\section{Experimental}

The ITO thin films were synthesized on soda lime glass substrates by an RF sputtering system, which is shown in Fig. 1. An RF sputtering system includes: a sputtering chamber that was evacuated to less than $9.4 \times 10^{-6}$ torr prior to sputter and then backfilled with argon gas and sputtering source (target) that was $3 \mathrm{in}$. diameter by $90 \% \mathrm{In}_{2} \mathrm{O}_{3}$ and $10 \% \mathrm{SnO}_{2}$ (99.99\% purity) in weight. The RF power and working pressure were $300 \mathrm{~W}$ and $2.2 \times 10^{-2}$ torr, respectively. The target distance from the substrate was about $7 \mathrm{~cm}$ and was pre-sputtered for at least $15 \mathrm{~min}$ to remove the contaminants on its surface. Deposition time was varied from 10 to $30 \mathrm{~min}$ and no oxygen was added during the growth. ITO thin films, having thicknesses of 100, 200 and $300 \mathrm{~nm}$, were synthesized on the soda lime glass substrate. The microstructures of the ITO thin films were investigated using $\mathrm{XRD}$ with $\mathrm{Cu}-\mathrm{K} \alpha$ radiation at a wavelength of $1.54 \AA$ and field emission scanning electron microscopy (FESEM). The morphology and root-mean-square surface roughness of the ITO thin films were obtained with AFM.

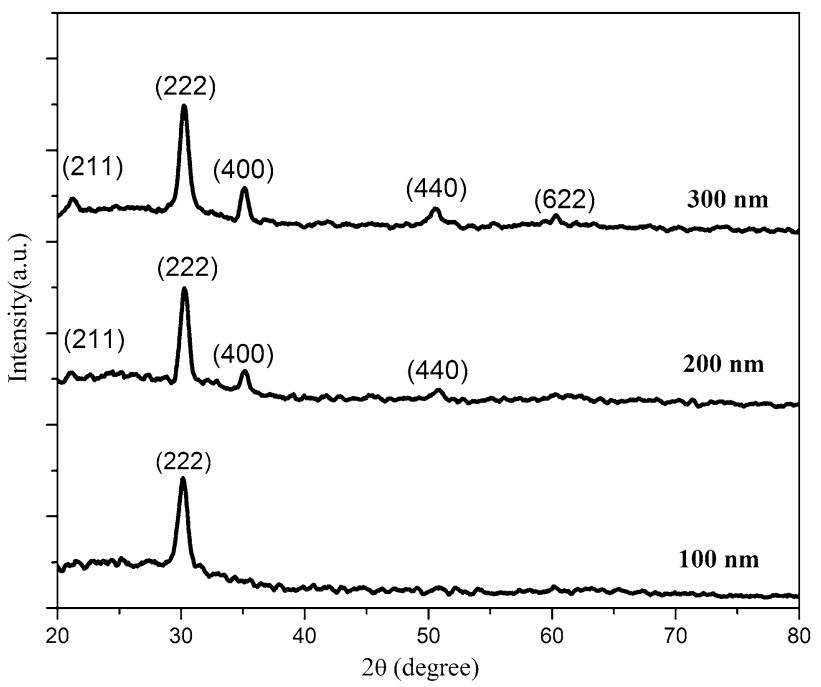

Fig. 2 X-ray diffraction patterns of 100, 200 and $300 \mathrm{~nm}$ ITO thin films grown at room temperature

\section{Result and discussion}

The XRD pattern of the ITO thin films for different thicknesses is shown in Fig. 2. For the film with $100 \mathrm{~nm}$ thickness, the (2 2 2) peak can be observed, and by increasing the thickness of the thin film to $200 \mathrm{~nm}$, the sample shows

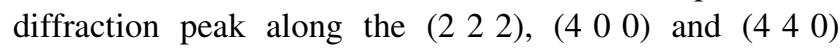

Fig. 1 Schematic of RF magnetron sputtering

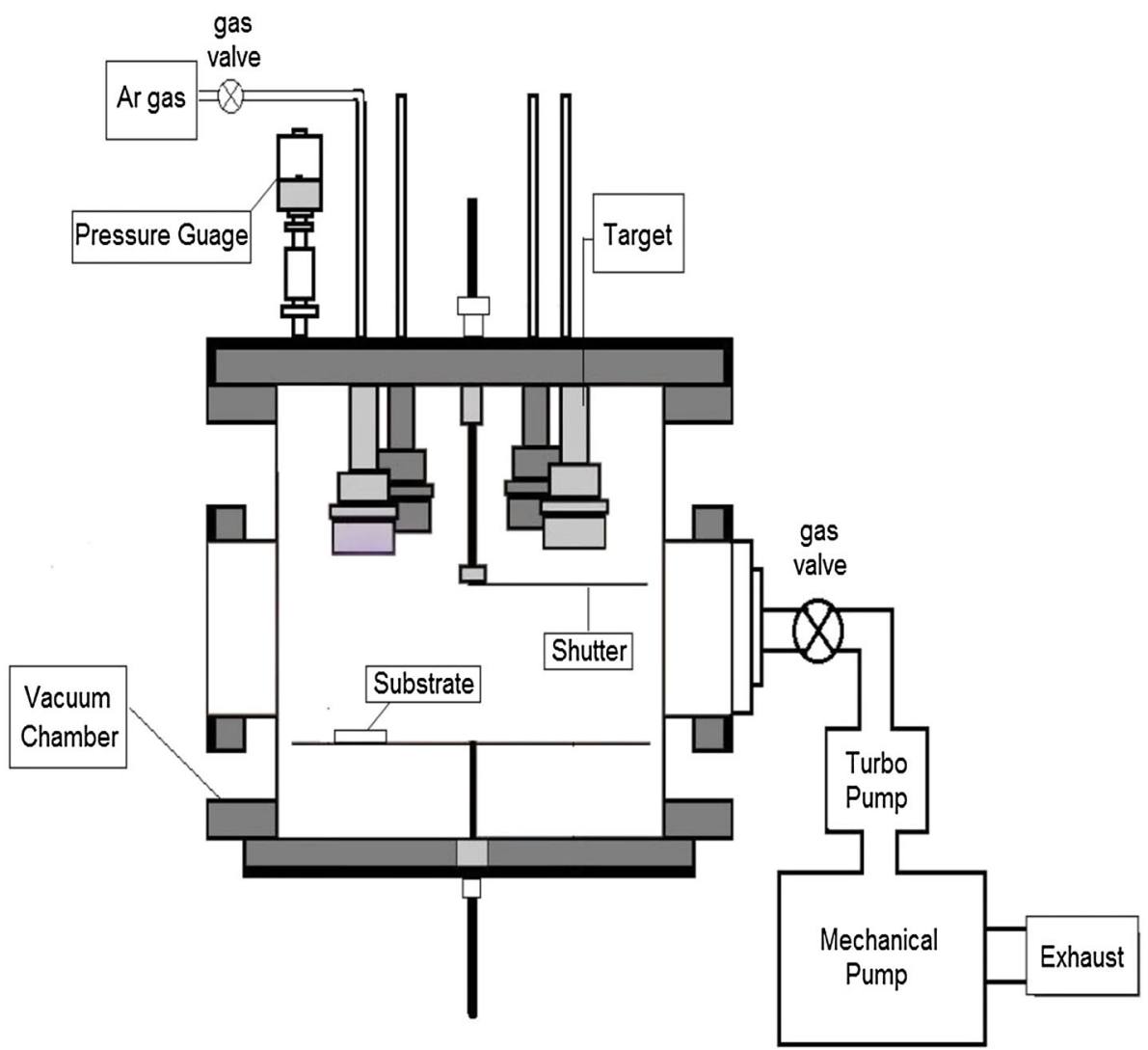


directions, where the (llll 22 2) peak has the preferred orientation (maximum intensity). Furthermore, by increasing the thickness to $300 \mathrm{~nm}$, additional diffraction peaks also appear, such as the formation of crystallites in the $\left(\begin{array}{lll}2 & 1 & 1\end{array}\right)$, (2 2 2 $)$, ( $\left(\begin{array}{lll}4 & 0 & 0\end{array}\right),\left(\begin{array}{lll}4 & 4 & 0\end{array}\right)$ and $\left(\begin{array}{lll}6 & 2 & 2\end{array}\right)$ orientations with a preferred orientation still along the (2 22 2) direction. These data are in good agreement with the reported values [21]. The peak is verified to be the (2 2 2) peak of cubic bixbyite $\mathrm{In}_{2} \mathrm{O}_{3}$. On the whole, we could report that a crystalline structure is observed for ITO film grown on glass prepared by RF magnetron sputtering at room temperature even when the thickness is $100 \mathrm{~nm}$. In fact, it is quite possible to acquire a crystalline structure even for the ITO film deposited at room temperature, if only the thickness is large enough. These results can help in the growth of TCO for use as a front contact in thin-film solar cells, which need to be thin in the order of $100 \mathrm{~nm}$ to keep high transmittance. Moreover, less resistivity is another important parameter for a TC, which is strongly affected by crystallinity.

In general, kinetic energies of sputtered particles enhance the surface migration of arriving particles at the substrate surface and the crystallinity of the films is greatly affected by them. Therefore, a polycrystalline structure could be grown by magnetron sputtering at room temperature. When the thickness of the samples is increased, the crystallinity and the mean grain size improved.
The crystallite size is obtained by using the Scherer formula [23]:

$t=\frac{k \lambda}{\beta \cos \theta}$,

where $\theta$ is the Bragg angle, $\beta$ is the full width at half maximum (FWHM in radians) of the peak corrected for instrumental broadening, $\lambda=0.154 \mathrm{~nm}$ is the wavelength of the X-ray and $k$ is the Scherer constant, its value being taken as 0.9 for calculations.

The calculated values of the crystalline sample sizes are shown in Table 1.

As seen, the thickness of the thin films is increased by increasing the deposition time. So by passing $30 \mathrm{~min}$ for growing the thin film, the thickness of the sample is reported to be about $300 \mathrm{~nm}$. On the other hand by increasing the deposition time, the crystal size is reduced from 25.440 to $17.226 \mathrm{~nm}$. Figure 3 shows the SEM morphologies for samples, which were deposited on soda lime glass substrates at room temperature. It can be observed that the films have not only a smooth surface, but also a form of a network of high-porosity interconnected nanoparticles, which approximately have a pore size ranging between 20 and $30 \mathrm{~nm}$. These results are consistent with X-ray measurements confirming that the thin films have high porosity and high surface area.

Table 1 Properties of the RF sputtering-grown ITO thin films on glass substrate for 10, 20 and 30 min deposition time

\begin{tabular}{llll}
\hline Sample & Deposition time $(\mathrm{min})$ & Thickness $(\mathrm{nm})$ & Crystal size $(\mathrm{nm})$ \\
\hline$a$ & 10 & 100 & 25.440 \\
$b$ & 20 & 200 & 21.919 \\
$c$ & 30 & 300 & 17.226 \\
\hline
\end{tabular}
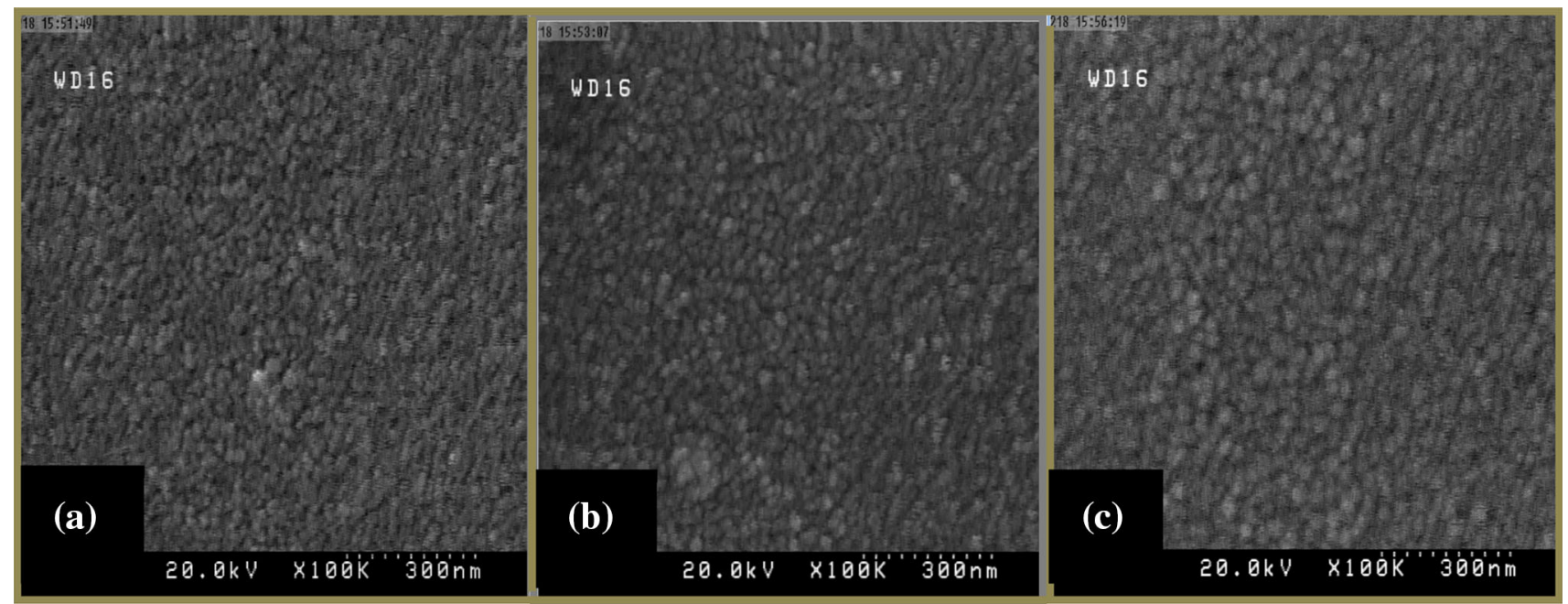

Fig. 3 SEM observations of the surfaces of ITO thin films. a $100 \mathrm{~nm}$, b $200 \mathrm{~nm}$ and c $300 \mathrm{~nm}$ 


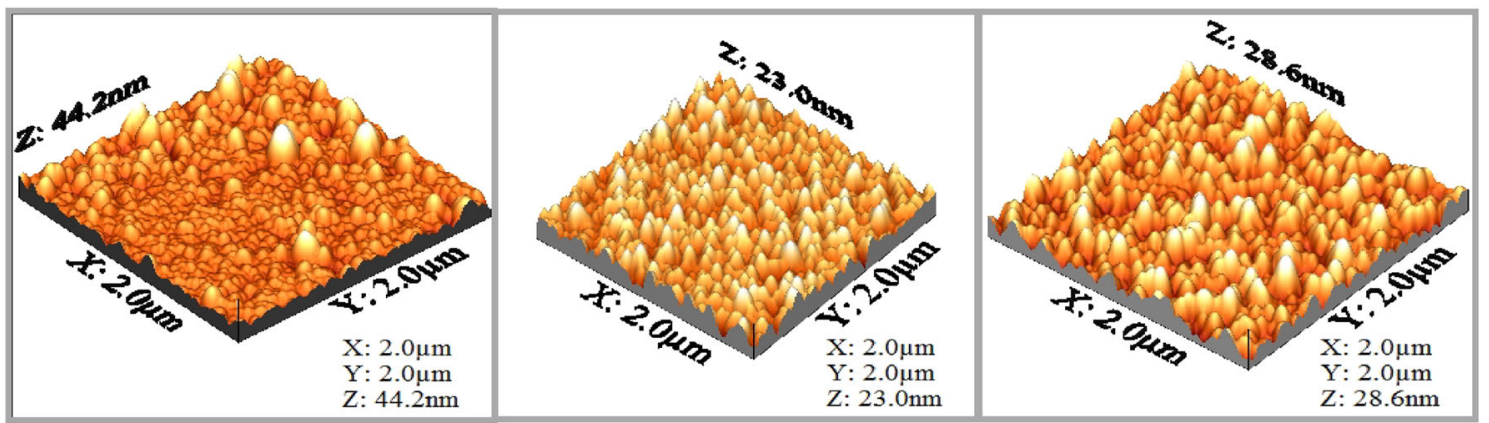

(a)

(b)

(c)

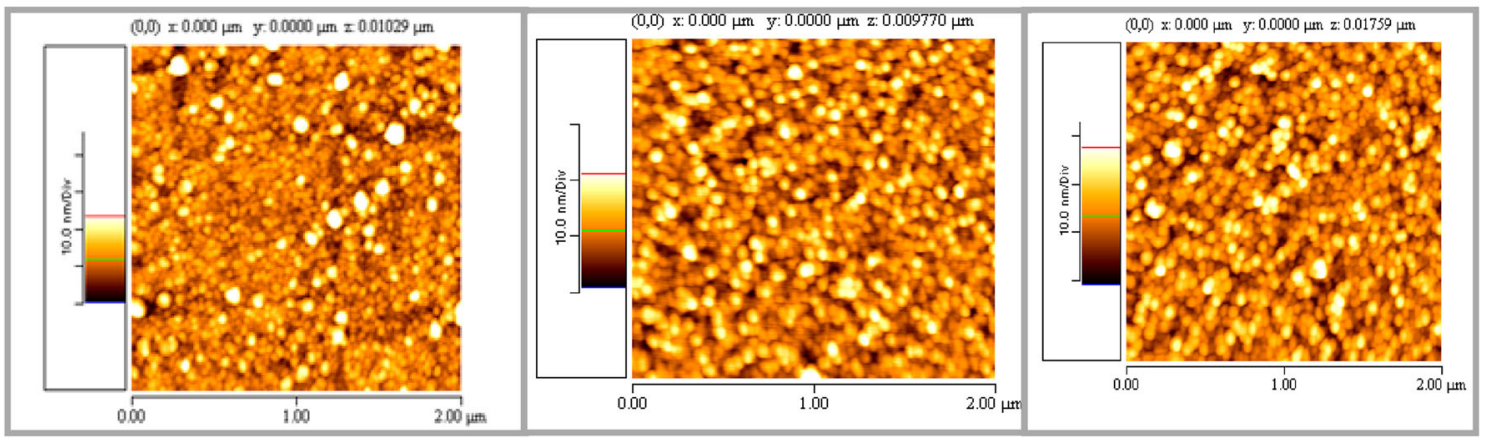

Fig. 4 3D and 2D AFM images of the ITO/glass samples deposited at thicknesses of a $100 \mathrm{~nm}, \mathbf{b} 200 \mathrm{~nm}$ and $\mathbf{c} 300 \mathrm{~nm}$
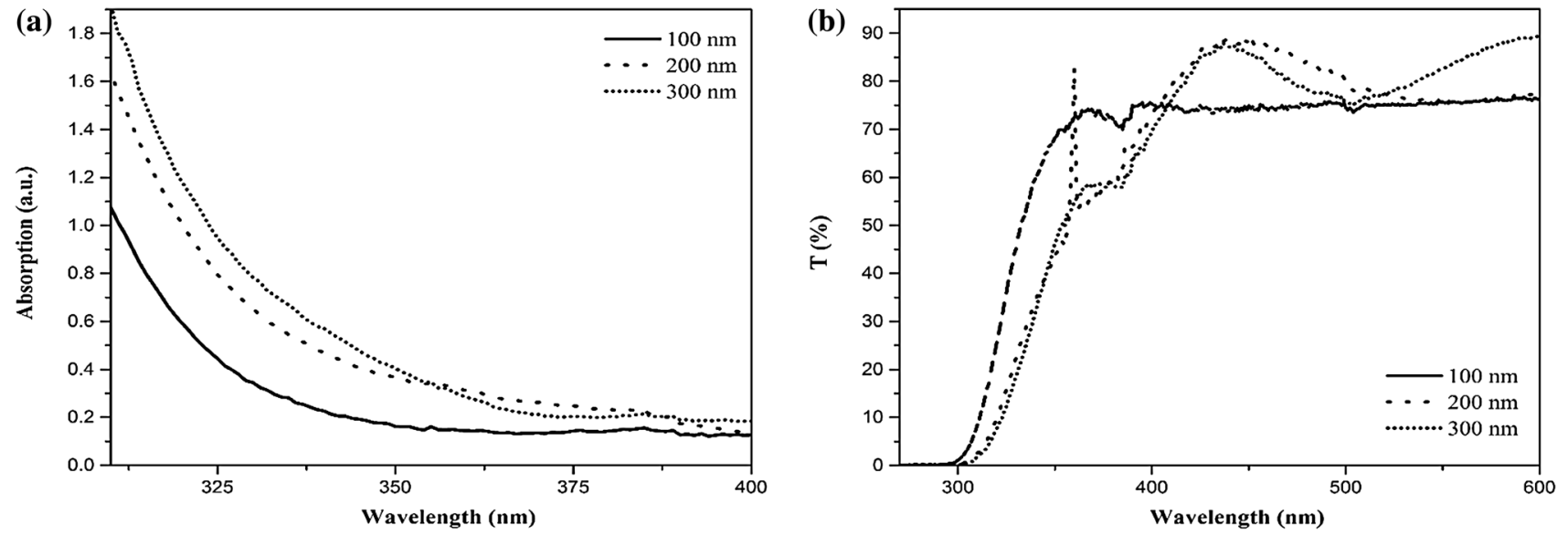

Fig. 5 a Transmission spectra and $\mathbf{b}$ absorption spectra of the ITO/glass samples deposited with different thicknesses of $100,200 \mathrm{and} 300 \mathrm{~nm}$

The surface morphology of ITO films for different thicknesses was investigated by atomic force microscopy (AFM). All the thin films have a smooth surface morphology (as anticipated due to the low power density), and Fig. 4 shows the AFM images (scan area $2 \times 2 \mu \mathrm{m}$ ) of the samples. Figure 4 illustrates the strong effect of growth time on surface morphology. A study of the AFM images (Fig. 4a) exhibits tiny grain growth by some bigger grains and claims that 10 min growth time cannot create a suitable uniform structure. By increasing thickness to $200 \mathrm{~nm}$ (Fig. 4b), small grains combine and form bigger grains and a columnar structure is observed. Eventually, at $30 \mathrm{~min}$ growth time (Fig. 4c), bigger grains for clusters and a dense and uniform structure. Therefore, the AFM study reveals that the grain size of the films clearly increases as the thickness increases. Also, the roughness of the ITO/glass thin films was studied. Surface roughness increases from 3.93 to $5.18 \mathrm{~nm}$, by increasing the film thickness from 100 to $300 \mathrm{~nm}$, which is a result of grain growth.

Resistivity values were calculated from sheet resistance measurements using a four-point probe. ITO films with thicknesses of 100, 200 and $300 \mathrm{~nm}$ were deposited onto 
glass substrates with a sheet resistance of $15.4 \times 10^{-4}$, $9.64 \times 10^{-4}$ and $2.43 \times 10^{-5} \Omega \mathrm{cm}$. There was a drastic improvement in the conductivity with increasing thickness.

It is clear that the electrical resistivity of the ITO films dramatically changes with the deposition time. This is because of the fact that, to get more uniform ITO films, the thickness should increase to have less impact of the roughness due to the substrate. One can conclude that the lower resistivity $2.43 \times 10^{-5} \Omega \mathrm{cm}$ at thickness $300 \mathrm{~nm}$ is the result of an improved crystallinity, high oxygen vacancy concentration and grain growth, which reduce grain boundary scattering and surface roughness.

Figure 5a, b illustrates the optical transmission and absorption data of the ITO thin films prepared with different thicknesses of 100, 200 and $300 \mathrm{~nm}$. In Fig. 5a, except for the sample with $100 \mathrm{~nm}$ thickness, the other two samples show interfering fringes at wavelength of $400 \mathrm{~nm}$ and more, which implies higher uniformity of the films. It is worth noting that red shift of the optical absorption edges is observed by increasing the film thickness from 100 to $300 \mathrm{~nm}$, while specifying the reduction in band-gap energy values. The average transmission of the films is about $80 \%$ which is similar to the previously reported data [24]. From Fig. 5b, which shows the absorption spectra of the ITO films with different thicknesses, the adsorption edges shift to higher wavelengths, in good agreement with the transmission data. The band-gap decrease can be due to the structural disorder or oxygen content in the crystalline lattice [25].

\section{Conclusion}

ITO thin films were grown by RF magnetron sputtering using Ar plasma without heating the substrate. The present study suggest that the ITO films structural and morphological properties depends on the film thickness which varies with deposition time. By increasing the deposition time, the thickness of the thin films is increased although the crystal size and electrical resistivity are reduced. The $\mathrm{X}$-ray diffraction data indicate polycrystalline thin films with grain orientations predominantly along the $\left(\begin{array}{lll}2 & 2 & 2\end{array}\right)$ and ( $\left.\begin{array}{lll}4 & 0 & 0\end{array}\right)$ directions. The FESEM images show that the thin films have a smooth surface and in the form of a network of high-porosity interconnected nanoparticles. The AFM images of the samples show that the roughness of the thin films is also increased due to the aggregation of grains by improving the crystallinity. The optical study of the ITO films reveals appropriate transparency, and optical results indicate that the grown ITO film is a good candidate for use as a TCO layer in optoelectronic devices.

Open Access This article is distributed under the terms of the Creative Commons Attribution 4.0 International License (http:// creativecommons.org/licenses/by/4.0/), which permits unrestricted use, distribution, and reproduction in any medium, provided you give appropriate credit to the original author(s) and the source, provide a link to the Creative Commons license, and indicate if changes were made.

\section{References}

1. Vasant Kumar, C.V.R., Mansingh, A.: Effect of target-substrate distance on the growth and properties of rf-sputtered indium tin oxide films. J. Appl. Phys. 65, 1270 (1989)

2. Knickerbocker, S.A., Kulkarni, A.K.: Estimation and verification of the optical properties of indium tin oxide based on the energy band diagram. J. Vac. Sci. Technol. A13(3), 1048 (1995)

3. Nisha, M., Anusha, S., Antony, A., Manoj, R., Jayaraj, M.K.: Effect of substrate temperature on the growth of ITO thin films. Appl. Surf. Sci. 252, 1430 (2005)

4. Akkad, F.E., Marafi, M., Punnoose, A., Prabu, G.: Effect of substrate temperature on the structural, electrical and optical properties of ITO films prepared by rf magnetron sputtering. Phys. Status Solidi 177, 445 (2000)

5. Wohlmuth, W., Adesida, I.: Properties of RF magnetron sputtered cadmium tin oxide and indium tin oxide thin films. Thin Solid Film 479, 223 (2005)

6. Reddy, V.S., Das, K., Dhar, A., Ray, S.K.: The effect of substrate temperature on the properties of ITO thin films for OLED applications. Semicond. Sci. Technol. 21, 1747 (2006)

7. Ali, H.M., Mohamed, H.A., Mohamed, S.H.: Effect of annealing on properties of $\mathrm{CuInS}_{2}$ thin films. Eur. Phys. J. Appl. Phys. 31, 87 (2005)

8. Liu, C., Mihara, T., Matsutani, T., Asanuma, T., Kiuchi, M.: Preparation and characterization of indium tin oxide films formed by oxygen ion beam assisted deposition. Solid State Commun. 126, 509 (2003)

9. Adurodija, F.O., Izumi, H., Ishihara, T., Yoshioka, H., Yamada, K., Matsuiand, H., Motoyama, M.: Highly conducting indium tin oxide (ITO) thin films deposited by pulsed laser ablation. Thin Solid Film 350, 79 (1999)

10. Adurodija, F.O., Bruning, R., Asia, I.O., Izumi, H., Ishihara, T., Yoshioka, H.: Effects of laser irradiation energy density on the properties of pulsed laser deposited ITO thin films. Appl. Phys. A 81, 953 (2005)

11. Sawada, M., Higuchi, M.: Electrical properties of ITO films prepared by tin ion implantation in In2O3 film. Thin Solid Film 317, 157 (1998)

12. Lee, H.C., Park, O.O.: Electron scattering mechanisms in indiumtin-oxide thin films: grain boundary and ionized impurity scattering. Vacuum 75, 275 (2004)

13. Kerkache, L., Layadi, A., Dogheche, E., Remiens, D.: Physical properties of RF sputtered ITO thin films and annealing effect. J. Phys. D. Appl. Phys. 39, 184 (2006)

14. Deng, W., Ohgi, T., Nejo, H., Fujita, D.: Development of conductive transparent indium tin oxide (ITO) thin films deposited by direct current (DC) magnetron sputtering for photon-STM applications. Appl. Phys. A 72, 595 (2001)

15. Qiao, Z., Latz, R., Mergel, D.: Thickness dependence of $\operatorname{In}_{2} \mathrm{O}_{3}: \mathrm{Sn}$ film growth. Thin Solid Film 466, 250 (2004)

16. Gao, M.Z., Job, R., Xue, D.S., Fahrner, W.R.: Thickness dependenceof resistivity and optical reflectance of ITO films. Chin. Phys. Lett. 25, 1380 (2008)

17. Hao, L., Diao, X., Xu, H., Gu, B., Wang, T.: Thickness dependence of structural, electrical and optical properties of indium tin oxide (ITO) films deposited on PET substrates. Appl. Surf. Sci. 254, 3504 (2008) 
18. Guillen, C., Herrero, J.: Influence of the film thickness on the structure, optical and electrical properties of ITO coatings deposited by sputtering at room temperature on glass and plastic substrates. Semicond. Sci. Technol. 23, 075002 (2008)

19. Kim, D.H., Park, M.R., Lee, H.J., Lee, G.H.: Thickness dependence of electrical properties of ITO film deposited on a plastic substrate by RF magnetron sputtering. Appl. Surf. Sci. 253, 409 (2006)

20. Kim, H., Horwitz, J.S., Kushto, G., Pique, A., Kafafi, Z.H., Gilmore, C.M., Chrisey, D.B.: Effect of film thickness on the properties of indium tin oxide thin films. J. Appl. Phys. 88, 6021 (2000)

21. Liu, C., Matsutani, T., Asanuma, T., Murai, K., Kiuchi, M., Alves, E., Reis, M.: Room-temperature growth of crystalline indium tin oxide films on glass using low energy oxygen ion beam assisted deposition. J. Appl. Phys. 93, 2262 (2003)
22. Kumar, K.J., Raju, N.R.C., Subrahmanyam, A.: Thickness dependent physical and photocatalytic properties of ITO thin films prepared by reactive DC magnetron sputtering. Appl. Surf. Sci. 257(7), 3075-3080 (2011)

23. Nair, P.K., Gomez-Daza, O., Readigos, A.A.C., Campos, J., Nair, M.T.S.: Formation of conductive CdO layer on CdS thin films during air heating. Semicond. Sci. Technol. 16(8), 651-656 (2001)

24. Heo, K.C., Sohn, Y., Gwag, J.S.: Effects of an additional magnetic field in ITO thin film deposition by magnetron sputtering. Ceram int 41, 617-621 (2015)

25. Bouzidi, A., Omri, K., El Mir Mabrouk, L., Guermazi, H.: Preparation, structural and optical investigations of ITO nanopowder and ITO/epoxy nanocomposites. Mater. Sci. Semicond. Process. 39, 536-543 (2015) 